\title{
Pressure Loss Coefficients for Asymmetric Bifurcations of Pulmonary Airways with Predetermined Flow Distributions
}

\author{
Alicia Clark ${ }^{1,2}$, Jenn S Rossmann ${ }^{1}$, Ira M Katz ${ }^{1,2 *}$, Andrew R Martin ${ }^{3}$ and Georges Caillibotte ${ }^{2}$ \\ ${ }^{1}$ Department of Mechanical Engineering, Lafayette College, Easton PA 18042, USA \\ ${ }^{2}$ Medical R \& D, Air Liquide International Health Research Centre Paris-Sac lay, 1 Chemin de la Porte des Loges, PO Box 126, Les Loges-en-Josas 78354, France \\ ${ }^{3}$ Department of Mechanical Engineering, University of Alberta, Edmonton AB T6G 2G8, Canada
}

\begin{abstract}
Computational Fluid Dynamics simulations of inspiratory airflow in asymmetric bifurcations have been performed in order to determine the influence of the asymmetry and Reynolds number on pressure losses over a physiologically relevant range for pulmonary airways; thus the results of this work can contribute to the understanding of respiratory ventilation in health and in disease. A key a priori insight to the design of the study is that the flow distribution in respiratory bifurcations can be largely independent of the local losses; and therefore, is predetermined by the boundary conditions in these calculations. The results, presented in the form of pressure loss coefficients, indicate that asymmetry and downstream conditions are significant for severe restrictions and laminar flow; but are relatively insignificant for turbulent flow conditions and for flow through the healthy branch.
\end{abstract}

Keywords: Pulmonary; Asymmetry; Airways; Loss coefficients; Flow distributions; CFD

\section{Introduction}

The distribution of inhaled air throughout human lungs is influenced in part by the pressure losses in lung airways. These losses are reflections of patient health; alterations in measured pressure drops can indicate the presence of respiratory disease [1]. The seminal work of Olson and colleagues [2] clearly demonstrated the contributions of various anatomical features to these energy losses, isolating the effects of branching angle and cross-sectional area on calculated pressure losses in pulmonary airways.

Pressure losses also have implications for the effective treatment of respiratory diseases, as they affect the extent to which inhaled medication is distributed throughout the lung. It is therefore of interest to examine the pressure losses within diseased airways. Heterogeneous airway obstructions are associated with a variety of respiratory diseases, including asthma, COPD, and cystic fibrosis, and can limit ventilation of obstructed regions of the lung [3-6].

Due to the complexity of multi-generational branching in the lungs, it is difficult to develop a single model that accurately and reliably predicts the flow of inhaled gas in all circumstances. Following the single-patient model of Weibel [7], researchers developing geometric models have had to choose whether to model one patient's airways accurately, or to simplify the morphology in order to generalize their results. Lung airway shape and structure vary significantly both within and among subjects $[8,9]$, the average branching angle varies from $15^{\circ}$ to $70^{\circ}[10]$, and the length-to-diameter ratio depends strongly on both generation and parent diameter [11]. Most previous airflow models have simplified the morphology of the lungs by assuming that each bifurcation is symmetric [12]; others have included more complexity, but have not modeled airway obstructions $[11,13]$.

It is the intention of the current work to use Computational Fluid Dynamics (CFD) to demonstrate and quantify the influence of asymmetry and bifurcation geometry on the pressure losses in branching airways, while recognizing that the ultimate flow distribution is largely determined by the particular placement of the bifurcation in the complex network of airways within the lung. For example, the flow distribution is often much more influenced by downstream lung volume or heterogeneous lung compliance than the asymmetry of a particular branch. Thus, a parametric analysis is performed where the morphologies and flow conditions are varied independently. Airway diameters are varied in such a way as to simulate the effect of natural asymmetry or the reduced lumen that can occur due to some respiratory diseases. The results in the form of pressure loss coefficients are provided and general observations are discussed that could inform the development of future airway fluid mechanics models.

\section{Methods}

\section{Numerical method}

The airflow through the lungs is governed by the Navier-Stokes equations, and the flow in any airway can be characterized by the nondimensional Reynolds number, Re:

$$
\operatorname{Re}=\frac{\rho v D}{\mu}
$$

Where $\rho$ and $\mu$ represent the air's density and viscosity, respectively; $\mathrm{v}$ is the mean velocity, and $\mathrm{D}$ is the diameter of the airway of interest. The air is assumed to have constant properties: density $\rho=1.225 \mathrm{~kg} / \mathrm{m}^{3}$ and viscosity $\mu=1.7894^{\star} 10-5 \mathrm{~kg} / \mathrm{s} \mathrm{m}$. In the current work, the reference Reynolds number is calculated using conditions at the inlet of the parent airway.

The transition from laminar to turbulent flow occurs around $\mathrm{Re}=2000$ in circular pipes. While respiratory flow is unsteady and

*Corresponding author: Ira Katz, Medical R \& D, Air Liquids Internationa Health Research Centre Paris -Sac lay, 1 Chemin de la Porte des Loges, PO Box 126, Les Loges-en-Josas 78354, France, Tel: +33 1390765 11; E-mail: Ira.katz@airliquide.com

Received: Febraury 28, 2015; Accepted: March 24, 2015; Published: March 31 2015

Citation: Clark A, Rossmann JS, Katz IM, Martin AR, Caillibotte G (2015) Pressure Loss Coefficients for Asymmetric Bifurcations of Pulmonary Airways with Predetermined Flow Distributions. J Bioengineer \& Biomedical Sci 5: 148 doi:10.4172/2155-9538.1000148

Copyright: ( $) 2015$ Clark A, et al. This is an open-access article distributed under the terms of the Creative Commons Attribution License, which permits unrestricted use, distribution, and reproduction in any medium, provided the original author and source are credited. 
changes direction from inspiration to exhalation, the current work considers only inspiratory flow, and examines steady flow at a range of Re values to examine the full range of physiological flow conditions. The three-dimensional bifurcation geometries of interest were meshed using GAMBIT (ANSYS, PA, USA), and the flow was simulated using the finite volume solver FLUENT (ANSYS, PA, USA). A mesh refinement study confirmed that the pressure drops and loss coefficients were sufficiently well resolved. A "boundary layer" mesh, six cells deep, was used at the walls to better quantify shear stresses, and to achieve grid independence of the solutions. The number of nodes and elements in the mesh varied depending on the diameter and length of the daughter vessels; the average number of elements for all models was 835,000 . Within FLUENT, second-order-accurate discretization schemes were used for all terms. Pressure-velocity coupling was achieved using the SIMPLE algorithm, and the transitional k-kl- $\omega$ model, a three-equation eddy-viscosity model for laminar and turbulent kinetic energies $(\mathrm{k}$ and $\mathrm{kl}$, respectively) as well as inverse turbulent time scale $(\omega)$, was incorporated. The model is based on two transport equations, one for intermittency and one for the transition onset criteria in terms of momentum thickness Reynolds number. The transport equations are intended for the implementation of correlation-based models into general-purpose CFD methods [14]. The theoretical framework and correlation constants for the turbulence model are beyond the scope of this paper but are provided in the FLUENT Theory Guide [15]. The Reynolds-Averaged Navier-Stokes (RANS) model devolves to the laminar Navier-Stokes equations when the turbulent fluctuations are negligible such that it was applicable for all of the cases considered, even those that remained laminar throughout.

The boundary conditions included no-slip and no-penetration at the walls; a prescribed uniform inlet velocity for the parent airway; and outlet conditions on the daughter vessels to simulate the influence of downstream effects in the pulmonary network. The outlet boundaries were given classic outflow conditions on downstream derivatives but included flow rate weighting to prescribe the distribution of inlet flow to the daughter vessels, such that each daughter received a specified portion of the parent vessel's volumetric airflow.

\section{Calculation of losses}

In addition to the mass and momentum conservation equations that govern flow, it is possible to consider the energy balance for steady, incompressible flow from any location $\mathrm{A}$ to location $\mathrm{B}$ along the airway:

$$
\left(\frac{p}{\rho}+\alpha \frac{v^{2}}{2}+g z\right)_{A}-\left(\frac{p}{\rho}+\alpha \frac{v^{2}}{2}+g z\right)_{B}=\sum h_{A-B},
$$

Where $\mathrm{p}$ and $\mathrm{v}$ are the average cross-section pressure and velocity at each station, and $\mathrm{z}$ is the position with respect to gravity g. The kinetic energy term is modified by a coefficient that varies to account for different flow profiles: $\alpha=1$ for a blunt velocity profile and $\alpha=2$ for parabolic. The term on the right hand side of equation (2), known as the head loss, represents the sum of all the resistive energy losses per unit mass as the flow moves from A to B. The energy balance in equation (2) assumes that there are no other sources of energy (heat transfer, or moving walls) present.

The head loss can also be expressed as the combination of major losses (for the straight parent and relevant daughter segments) and minor losses $\mathrm{K}$ (for the bifurcation):

$$
\sum h_{A-B}=\left(f \frac{L}{D} \frac{v^{2}}{2}\right)_{\text {parent }}+\left(f \frac{L}{D} \frac{v^{2}}{2}\right)_{\text {daughter }}+K_{\text {bifurcation }}
$$

Here $\mathrm{f}$ is a friction factor whose value depends on the flow regime and Reynolds number Re. For simplicity we will refer to the loss coefficient $\mathrm{K}$ without the subscript because it is the only one considered herein. For laminar, fully developed flow, $\mathrm{f}$ is analytically determined:

$$
f=\frac{64}{\operatorname{Re}}
$$

For turbulent flow, $\mathrm{f}$ is calculated from the Blasius correlation:

$$
f=\frac{0.316}{\operatorname{Re}^{0.25}}
$$

It should be noted that the energy balance of equation (2) is applied from location A along the parent vessel to a station B on either daughter vessel, so that there are two values of head loss associated with a single bifurcation. These values will be equal if the bifurcation is completely symmetric, but in the case of asymmetric bifurcation, there will be two values of $h_{A-B}$ and, accordingly, two values of $K$. We will use the notation "left" and "right" to distinguish between the loss coefficients for the two daughter vessels.

The desired coefficient $\mathrm{K}$ for either daughter vessel can be isolated by rearranging the previous two expressions.

$$
K=\frac{\frac{p_{\text {enty }(A)}-p_{\text {exit }(B)}}{\rho}+\left(\alpha \frac{v^{2}}{2}\right)_{\text {enty }(A)}-\left(\alpha \frac{v^{2}}{2}\right)_{\text {exir }(B)}-\left(f \frac{L}{D} \frac{v^{2}}{2}\right)_{\text {parent }}-\left(f \frac{L}{D} \frac{v^{2}}{2}\right)_{\text {daugher }}}{\frac{v_{\text {enty }}^{2}(A)}{2}}
$$

\section{Model geometry}

To explore flow patterns over the full range of flow rates and inhaled gases for many generations of airways of the human tracheobronchial system would require an immense effort; herein a single base model was employed where the inlet $\mathrm{Re}$ was varied from $\mathrm{Re}=100$ (which occurs in lower regions of the lung) to $\mathrm{Re}=2000$ (which occurs nearer the trachea) [16]. A schematic is shown in Figure 1. For the base model the diameter of the parent vessel was held at $0.01 \mathrm{~m}$ and the non-restricted right daughter at $0.008 \mathrm{~m}$ for all simulations, while the diameter of the left daughter branch was varied to explore the effects of downstream constriction. It should be emphasized that while FLUENT requires the input of particular dimensional data, the reformulated inputs and outputs as discussed below are applicable throughout the

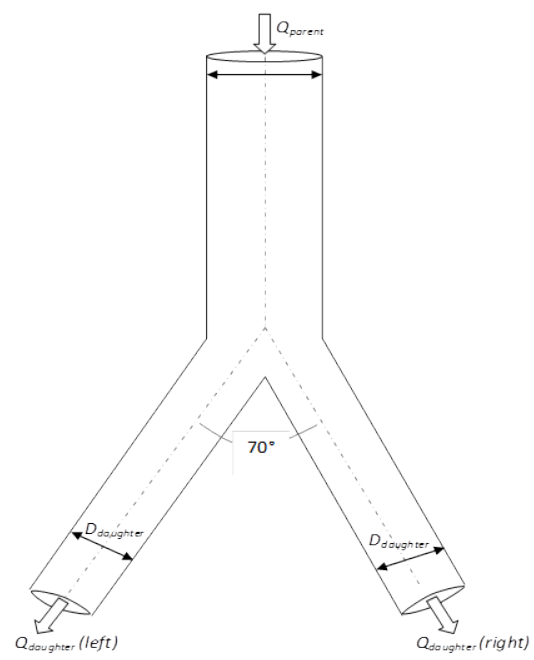

Figure 1: Schematic showing base geometry of single bifurcation. Vesse and fluid parameters are adjusted to make airflow through this geometry representative of a wide range of tracheobronchial flows. 
lung. The remaining geometric parameters were held constant. For example, the bifurcation angle is fixed at $70^{\circ}$, with each daughter vessel having a branching angle of $35^{\circ}$ from the parent vessel's mid plane and the inlet and outlet lengths where 20D to allow for flow development .

As indicated above, the effects of disease on flow in the lungs are modeled by flow boundary conditions and by changes to the geometric model. Three key non-dimensional parameters are varied independently: the Reynolds number Re, defined in equation (1); and the ratios of daughter to parent volumetric flow rates (equation 7) and diameters (equation 8). The volumetric flow rate ratio $\mathrm{Q}_{\text {ratio }}$ is expressed:

$$
Q_{\text {ratio }}=\frac{Q_{\text {daughter }}}{Q_{\text {parent }}}
$$

Where $\mathrm{Q}_{\text {daughter }}$ represents the left daughter branch's proportion of the inlet flow; the flow rate in the right daughter vessel is prescribed to be $1-Q_{\text {daughter }}$. The value of $Q_{\text {ratio }}$ is permitted to vary from 0.1 to 1 to simulate a range of disease states. The ratio of daughter to parent vessel diameters is:

$$
D_{\text {ratio }}=\frac{D_{\text {daughter }}}{D_{\text {parent }}}
$$

Where $\mathrm{D}_{\text {daughter }}$ represents the left daughter vessel, as the right daughter vessel diameter is held fixed (at $0.8 \mathrm{D}_{\text {parent }}$ ) throughout the study. $\mathrm{D}_{\text {ratio }}$ is varied from 0.4 to 1.0 . The entry and exit terms of Equation 6 were taken at one diameter upstream $(\mathrm{L} / \mathrm{D}=-1)$ and 19 diameters downstream $(L / D=19)$ of the bifurcation to allow for fully developed flow. In all, 100 CFD simulations were performed (Table 1) with two $K$ values being calculated per simulation, one for the left (diseased) branch and one for the right (healthy) branch.

\section{Results and Discussion}

The calculated flow patterns are consistent with previous studies of flow in bifurcations [13,17-19]. Flow separation occurs in some cases, with the imposed flow distribution $\left(\mathrm{Q}_{\text {ratio }}\right)$ being the strongest predictor of separation. This is seen in Figures 2 and 3: in Figure 2, a pathological $\mathrm{Q}_{\text {ratio }}$ of 0.8 results in significant separation in the right daughter vessel; in Figure 3, a $\mathrm{Q}_{\text {ratio }}$ of 0.3 yields low pressure but no backflow or separation in the same region.

The pressure loss coefficients in the form of $\mathrm{K}$ values for the left (diseased) and right (healthy) branches are given in Table 1 in the Supplementary Material. Some general comments can be made. The left (diseased) branch $\mathrm{K}$ values increase significantly when flow is shunted from the right (healthy) branch. For example, for $\mathrm{D}_{\text {ratio }}=0.4$ (a $50 \%$ reduction in diameter from the base case) and $\mathrm{Re}=100$, the $\mathrm{K}$ value increases over $400 \%$ (from 5.2 to 28.2 ) as the $\mathrm{Q}_{\text {ratio }}$ increases from 0.5 (symmetric) to 1.0 (totally shunted). However, this is the case only for laminar flow. Consider the equivalent case $\left(D_{\text {ratio }}=0.4, Q_{\text {ratio }}\right.$ increases from 0.5 to 1.0 ) with $R e=2000$; here the $K$ value actually decreases from 2.4 to 1.0 (but note that the overall pressure drop still increases). Another example where the presence of turbulence appears to dampen the increase in $K$ value can be observed by comparing simulations 18-20 where the parent $\mathrm{Re}=2000$. $\mathrm{K}$ values increase only about $40 \%$ (from 7 to 10.1). But note that there is a relative maximum because for $\mathrm{Q}_{\text {ratio }}=0.8$ $\mathrm{K}=16.1$. This can be explained by the fact that for the daughter $\mathrm{Re}=1993$ and 2492 (i.e., the Re straddles the turbulent limit) for $\mathrm{Q}_{\text {ratio }}=0.8$ and 1.0 , respectively; i.e., the presence of turbulence results in a relatively lower and more uniform value independent of the other parameters.

Over the full parameter range examined the $\mathrm{K}$ values for the right (healthy) daughter are relatively uniform (average of 1.3, standard

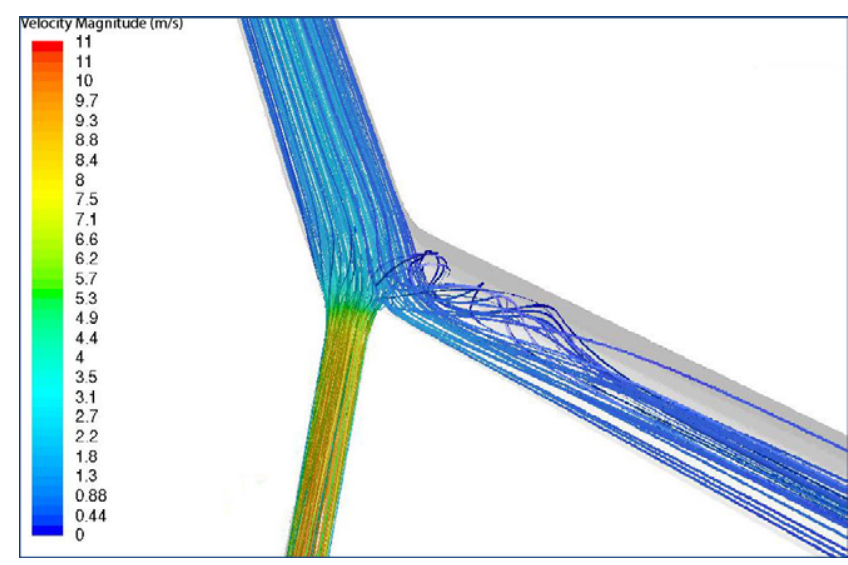

Figure 2: Calculated streamlines for parent $\mathrm{Re}=1000$, daughter diameter ratio $D_{\text {ratio }}=0.4$, with obstruction model forcing $80 \%$ of flow through the left daughter vessel $\left(Q_{\text {ratio }}=0.8\right)$. Branching angle is $70^{\circ}$. Streamlines are colored by velocity magnitude $(\mathrm{m} / \mathrm{s})$.
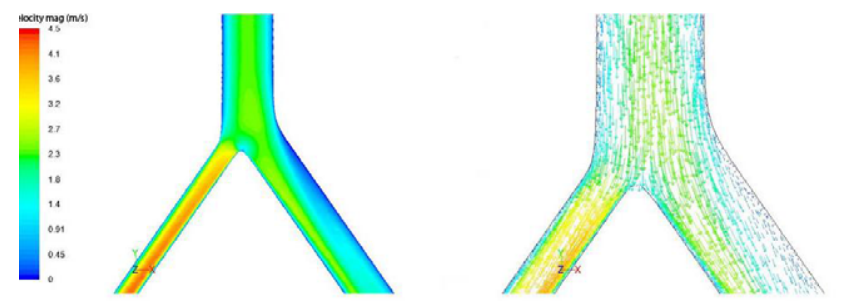

Figure 3: Calculated velocity magnitude contours (left) and vectors (right) for parent $\mathrm{Re}=1000$, daughter diameter ratio Dratio $=0.4$, with model forcing $30 \%$ of flow through the left daughter vessel, or Qratio $=0.3$. Branching angle is $70^{\circ}$. Velocity vectors are colored byvelocity magnitude $(\mathrm{m} / \mathrm{s})$.

deviation of 0.4 ) and thus close to the symmetric cases (Simulation Nos. 53, 58, 63, 68, 73). A similar observation as discussed above can be made for the left (diseased) daughter for $\mathrm{R}=2000$ (average of 1.2, standard deviation of 0.5$)$. In fact, even for the laminar cases $(\mathrm{Re}=100$, 200,500 and 1000) when the flow is shunted away from the diseased to the healthy branch $\left(\mathrm{Q}_{\text {ratio }}=0.1,0.3\right.$ and 1.0$)$ the $\mathrm{K}$ values average 1.6, though the standard deviation is somewhat greater, 4.7. These results suggest that for a wide range of cases the effects of downstream conditions and asymmetry are relatively negligible and the symmetric values can most likely be employed giving reasonable results. However, for more severe restrictions in laminar flow downstream conditions should be taken into account.

We attempted without success to find analytical correlations for these results to make them easier to apply directly into models of airway pressure loss. However, given the observations stated above, perhaps limited correlations for only laminar flow and severe disease may be found that have adequate accuracy.

The CFD simulations performed in the current work reflect physiological conditions, and do not prescribe fully developed flow. The resulting pressure drops are compared with those calculated using fully developed flow models for three representative cases in Figure 4. Although fully developed assumptions are implicit in the pressure drops and loss coefficients generally used in large network models, the differences shown in Figure 4 indicate that for shorter daughter sections that typically occur in the lung the inertial loss due to the bifurcation 

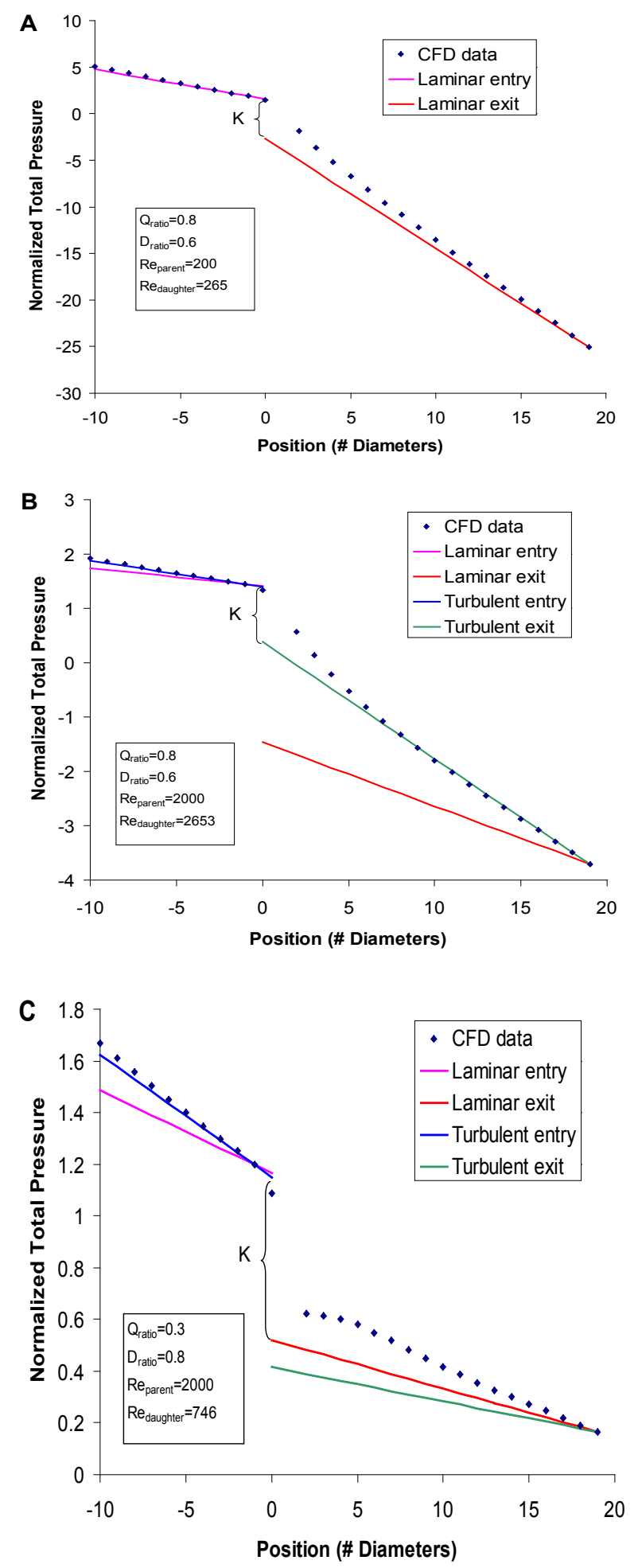

Figure 4: Pressure distributions through the parent and left diseased branch obtained from CFD, shown with pressure drops obtained from analytical, fully developed models to define the loss coefficient, for three representative cases: A) laminar entry and exit flow, B) turbulent entry and exit flow, and C) turbulent entry and laminar exit flow indicated by the reduced slope of the pressure loss downstream of the bifurcation. In panels $B$ and $C$ the analytica result for both fully developed laminar and turbulent flows are shown for comparison. Note that positions in the parent are taken as negative. will be somewhat overestimated. For example, for the cases illustrated in Panels A, B and C of Figure 4, if the daughter branch ended at 5 diameters instead of 19 as indicated the errors would be $23 \%, 9 \%$, and $30 \%$, respectively.

\section{Conclusion}

Pressure loss coefficients have been provided for asymmetric lung bifurcations over a physiological relevant range of incoming Reynolds number, the diameter ratio of parent to daughter, and the flow ratio. The flow ratio was prescribed to account for the fact that individual bifurcations are part of complex lung airway networks.

\section{Acknowledgment}

Lafayette College supported the first author as an undergraduate research assistant on this project. The authors also gratefully acknowledge the support of the NSF through grant CTS-0618923

\section{References}

1. Grotberg JB (2006) Dynamics of gas flow and pressure-flow relationships. In Laurent GJ, Shapiro SD, eds. Encyclopedia of Respiratory Medicine, Elsevier.

2. Olson DE, Dart GA, Filley GF (1970) Pressure drop and fluid flow regime of air inspired into the human lung. J Appl Physiol 28: 482-494.

3. De Lange EE, Altes TA, Patrie JT, Battiston JJ, Juersivich AP, et al. (2009) Changes in Regional Airflow Obstruction over Time in the Lungs of Patients with Asthma: Evaluation with 3He MR Imaging. Radiology 250: 567-575

4. Venegas JG, Winkler T, Musch G, Vidal Melo MF, Layfield D, et al. (2005) Self-organized patchiness in asthma as a prelude to catastrophic shifts. Nature 434: $777-782$.

5. Venegas JG, Schroeder T, Harris S, Winkler RT, Melo MFV (2005) The distribution of ventilation during bronchoconstriction is patchy and bimodal: A PET imaging study. Respir Physiol Neurobiol 148: 57-64

6. Ley-Zaporozhan J, Ley S, Kauczor HU (2007) Morphological and functiona imaging in COPD with CT and MRI: present and future. Eur Radiol 18: 510-521.

7. Weibel ER (1963) Geometry and Dimensions of Airways of Conductive and Transitory Zones. In: Morphometry of the Human Lung. Springer Berlin Heidelberg 1963: 110-135.

8. Choi J, Tawhai MH, Hoffman EA, Lin CL (2009) on intra and inter subject variability's of airflow in the human lungs. Phys Fluids 21: 101901.

9. Horsfield K, Dart G, Olson DE, Filley GF, Cumming G (1971) Models of the human bronchial tree. J Appl Physiol 31: 207-217.

10. Phalen RF, Yeh HC, Schum GM, Raabe OG (1978) Application of an idealized model to morphometry of the mammalian tracheobronchial tree. Anat Rec 190 167-176.

11. Phillips CG, Kaye SR (1995) Diameter-based analysis of the branching geometry of four mammalian bronchial trees. Respir Physiol 102: 303-316.

12. Kang MY, Hwang J, Lee JW (2011) Effect of geometric variations on pressure loss for a model bifurcation of the human lung airway. J Biomech 44: 1196-1199.

13. Liu Y, So RMC, Zhang $\mathrm{CH}$ (2002) Modeling the bifurcating flow in a human lung airway. J Biomech 35: 465-473.

14. Menter FR, Langtry RB, Likki SR, Suzen YB, Huang PG, et al. (2006) A correlation-based transition model using local variables-Part I: mode formulation. J Turbo machinery 128: 413-22.

15. Ansys (2011) Fluent Theory Guide-14.

16. Finlay WH (2001) The Mechanics of Inhaled Pharmaceutical Aerosols: An Introduction. Academic Press, London.

17. Zhang Z, Kleinstreuer C (2004) Airflow structures and nano-particle deposition in a human upper airway model. J Comput Phys 198: 178-210.

18. Zhang CH, Liu Y, So RMC, Phan-Thien N (2002) The influence of inlet velocity profile on three-dimensional three-generation bifurcating flows. Comput Mech 29: $422-429$

19. Zhang Z, Kleinstreuer C, Kim CS (2000) Flow Structure and Particle Transport in a Triple Bifurcation Airway Model. J Fluids Eng 123: 320-330. 
Citation: Clark A, Rossmann JS, Katz IM, Martin AR, Caillibotte G (2015) Pressure Loss Coefficients for Asymmetric Bifurcations of Pulmonary Airways with Predetermined Flow Distributions. J Bioengineer \& Biomedical Sci 5: 148. doi:10.4172/2155- 9538.1000148

Page 5 of 6

\section{Supplementary Material}

Table 1 provides the loss coefficients for each daughter airway for the 100 simulated cases

\begin{tabular}{|c|c|c|c|c|c|}
\hline $\begin{array}{l}\text { Simulation } \\
\text { No. }\end{array}$ & $D_{\text {ratio }}\left(D_{\text {left }} / D_{\text {parent }}\right)$ & $\operatorname{Re}$ & $\mathbf{Q}_{\text {ratio }}$ & $\mathrm{K}_{\text {left }}$ (diseased) & $\mathrm{K}_{\text {right }}$ (healthy) \\
\hline 1 & 0.4 & \multirow{5}{*}{100} & 0.1 & 0.5 & 2.4 \\
\hline 2 & 0.4 & & 0.3 & 1.2 & 1.6 \\
\hline 3 & 0.4 & & 0.5 & 5.2 & 1.2 \\
\hline 4 & 0.4 & & 0.8 & 16.9 & 1.2 \\
\hline 5 & 0.4 & & 1 & 28.2 & NA \\
\hline 6 & 0.4 & \multirow{5}{*}{200} & 0.1 & 0.8 & 2.3 \\
\hline 7 & 0.4 & & 0.3 & 2.5 & 1.6 \\
\hline 8 & 0.4 & & 0.5 & 7.1 & 1.2 \\
\hline 9 & 0.4 & & 0.8 & 19.1 & 1.1 \\
\hline 10 & 0.4 & & 1 & 30.1 & NA \\
\hline 11 & 0.4 & \multirow{5}{*}{500} & 0.1 & 1.0 & 2.2 \\
\hline 12 & 0.4 & & 0.3 & 3.1 & 1.6 \\
\hline 13 & 0.4 & & 0.5 & 7.6 & 1.2 \\
\hline 14 & 0.4 & & 0.8 & 18.4 & 1.1 \\
\hline 15 & 0.4 & & 1 & 28.1 & NA \\
\hline 16 & 0.4 & \multirow{5}{*}{1000} & 0.1 & 1.1 & 1.9 \\
\hline 17 & 0.4 & & 0.3 & 3.0 & 1.4 \\
\hline 18 & 0.4 & & 0.5 & 7.0 & 1.1 \\
\hline 19 & 0.4 & & 0.8 & 16.1 & 1.1 \\
\hline 20 & 0.4 & & 1 & 10.1 & NA \\
\hline 21 & 0.4 & \multirow{5}{*}{2000} & 0.1 & 1.0 & 0.8 \\
\hline 22 & 0.4 & & 0.3 & 2.7 & 1.1 \\
\hline 23 & 0.4 & & 0.5 & 2.4 & 0.9 \\
\hline 24 & 0.4 & & 0.8 & 1.8 & 1.0 \\
\hline 25 & 0.4 & & 1 & 1.0 & NA \\
\hline 26 & 0.6 & \multirow{5}{*}{100} & 0.1 & 1.0 & 2.1 \\
\hline 27 & 0.6 & & 0.3 & 0.9 & 1.4 \\
\hline 28 & 0.6 & & 0.5 & 1.7 & 1.0 \\
\hline 29 & 0.6 & & 0.8 & 4.3 & 1.0 \\
\hline 30 & 0.6 & & 1 & 6.9 & NA \\
\hline 31 & 0.6 & \multirow{5}{*}{200} & 0.1 & 1.0 & 2.2 \\
\hline 32 & 0.6 & & 0.3 & 1.0 & 1.4 \\
\hline 33 & 0.6 & & 0.5 & 1.9 & 1.0 \\
\hline 34 & 0.6 & & 0.8 & 4.6 & 1.0 \\
\hline 35 & 0.6 & & 1 & 7.2 & NA \\
\hline 36 & 0.6 & \multirow{5}{*}{500} & 0.1 & 1.0 & 2.2 \\
\hline 37 & 0.6 & & 0.3 & 1.1 & 1.5 \\
\hline 38 & 0.6 & & 0.5 & 2.0 & 1.1 \\
\hline 39 & 0.6 & & 0.8 & 4.4 & 1.0 \\
\hline 40 & 0.6 & & 1 & 6.8 & NA \\
\hline
\end{tabular}

\begin{tabular}{|c|c|c|c|c|c|}
\hline 41 & 0.6 & \multirow{5}{*}{1000} & 0.1 & 1.0 & 1.9 \\
\hline 42 & 0.6 & & 0.3 & 1.1 & 1.3 \\
\hline 43 & 0.6 & & 0.5 & 1.8 & 1.0 \\
\hline 44 & 0.6 & & 0.8 & 3.8 & 1.0 \\
\hline 45 & 0.6 & & 1 & 5.8 & NA \\
\hline 46 & 0.6 & \multirow{5}{*}{2000} & 0.1 & 0.9 & 0.8 \\
\hline 47 & 0.6 & & 0.3 & 1.0 & 1.0 \\
\hline 48 & 0.6 & & 0.5 & 1.5 & 0.9 \\
\hline 49 & 0.6 & & 0.8 & 1.2 & 1.0 \\
\hline 50 & 0.6 & & 1 & 1.4 & NA \\
\hline 51 & 0.8 & \multirow{5}{*}{100} & 0.1 & 0.9 & 2.0 \\
\hline 52 & 0.8 & & 0.3 & 0.8 & 1.2 \\
\hline 53 & 0.8 & & 0.5 & 0.9 & 0.9 \\
\hline 54 & 0.8 & & 0.8 & 1.6 & 0.8 \\
\hline 55 & 0.8 & & 1 & 2.5 & NA \\
\hline 56 & 0.8 & \multirow{5}{*}{200} & 0.1 & 1.0 & 2.1 \\
\hline 57 & 0.8 & & 0.3 & 0.8 & 1.4 \\
\hline 58 & 0.8 & & 0.5 & 1.0 & 1.0 \\
\hline 59 & 0.8 & & 0.8 & 1.7 & 0.9 \\
\hline 60 & 0.8 & & 1 & 2.6 & NA \\
\hline 61 & 0.8 & \multirow{5}{*}{500} & 0.1 & 1.0 & 2.2 \\
\hline 62 & 0.8 & & 0.3 & 0.9 & 1.5 \\
\hline 63 & 0.8 & & 0.5 & 1.1 (use 1.0) & 1.0 \\
\hline 64 & 0.8 & & 0.8 & 1.8 & 0.9 \\
\hline 65 & 0.8 & & 1 & 2.7 & NA \\
\hline 66 & 0.8 & \multirow{5}{*}{1000} & 0.1 & 1.0 & 1.9 \\
\hline 67 & 0.8 & & 0.3 & 0.9 & 1.3 \\
\hline 68 & 0.8 & & 0.5 & 1.0 & 1.0 \\
\hline 69 & 0.8 & & 0.8 & 1.6 & 0.9 \\
\hline 70 & 0.8 & & 1 & 2.3 & NA \\
\hline 71 & 0.8 & \multirow{5}{*}{2000} & 0.1 & 1.0 & 0.8 \\
\hline 72 & 0.8 & & 0.3 & 0.8 & 1.0 \\
\hline 73 & 0.8 & & 0.5 & 0.8 & 0.8 \\
\hline 74 & 0.8 & & 0.8 & 1.2 & 0.9 \\
\hline 75 & 0.8 & & 1 & 0.9 & NA \\
\hline 76 & 1 & \multirow{5}{*}{100} & 0.1 & 0.9 & 1.8 \\
\hline 77 & 1 & & 0.3 & 0.7 & 1.1 \\
\hline 78 & 1 & & 0.5 & 0.7 & 0.8 \\
\hline 79 & 1 & & 0.8 & 0.9 & 0.7 \\
\hline 80 & 1 & & 1 & 1.2 & NA \\
\hline 81 & 1 & \multirow{5}{*}{200} & 0.1 & 1.0 & 2.1 \\
\hline 82 & 1 & & 0.3 & 0.8 & 1.4 \\
\hline 83 & 1 & & 0.5 & 0.8 & 0.9 \\
\hline 84 & 1 & & 0.8 & 1.0 & 0.8 \\
\hline 85 & 1 & & 1 & 1.4 & NA \\
\hline
\end{tabular}


Citation: Clark A, Rossmann JS, Katz IM, Martin AR, Caillibotte G (2015) Pressure Loss Coefficients for Asymmetric Bifurcations of Pulmonary Airways with Predetermined Flow Distributions. J Bioengineer \& Biomedical Sci 5: 148. doi:10.4172/2155- 9538.1000148

\begin{tabular}{|c|c|c|c|c|c|}
\hline 86 & 1 & \multirow{5}{*}{500} & 0.1 & 1.0 & 2.3 \\
\hline 87 & 1 & & 0.3 & 0.9 & 1.5 \\
\hline 88 & 1 & & 0.5 & 0.9 & 1.0 \\
\hline 89 & 1 & & 0.8 & 1.1 & 0.9 \\
\hline 90 & 1 & & 1 & 1.5 & NA \\
\hline 91 & 1 & \multirow{5}{*}{1000} & 0.1 & 1.0 & 2.0 \\
\hline 92 & 1 & & 0.3 & 0.9 & 1.4 \\
\hline 93 & 1 & & 0.5 & 0.9 & 1.0 \\
\hline 94 & 1 & & 0.8 & 1.0 & 0.9 \\
\hline 95 & 1 & & 1 & 1.3 & NA \\
\hline 96 & 1 & \multirow{5}{*}{2000} & 0.1 & 1.0 & 0.9 \\
\hline 97 & 1 & & 0.3 & 0.9 & 1.1 \\
\hline 98 & 1 & & 0.5 & 0.8 & 0.8 \\
\hline 99 & 1 & & 0.8 & 0.8 & 0.8 \\
\hline 100 & 1 & & 1 & 1.1 & NA \\
\hline
\end{tabular}

